\title{
ALGO MÁS SOBRE LA EXPLICACIÓN
}

Margarita Ponce

InstTTUTO DE InVESTIGACIONES FILOSG FICAS Universidad Nacional autónoma de Mḱxico

\section{Introducción}

Con el interés de averiguar si la explicación funcional - una subclase de la teleológica- es una explicación genuina, como trasfondo en este trabajo, argumentaré ahora en favor de las tesis siguientes: $i$ ) explicar es una operación distinta de la de informar, describir o enumerar, y ii) toda explicación constituye, en último término, la respuesta a una pregunta "¿por qué?", sin que esto implique que dicha pregunta inquiera siempre por las causas del hecho o del fenómeno que deseamos explicar. Asimismo intentaré mostrar que la confusión entre las operaciones mencionadas en (i) surge, por una parte, de desligar las explicaciones de la inteligibilidad, de la comprensión, y, por otra, de creer que el objetivo primario de ellas es justificar la verdad del explanandum o proporcionar nuevos datos sobre el mundo. Para esto expondré, de manera breve, las ideas que son el antecedente de las que aquí sostengo; ilustraré luego la confusión entre explicar e informar, con el análisis de G. A. Cohen sobre la naturaleza de la explicación -propuesto en el capítulo IX de su libro Karl Marx's Theory of History (1978)-; y, para destacar la diferencia entre tales operaciones, recurriré a la caracterización de un episodio explicativo hecha por Sylvain Bromberger -en "An Approach to Explanation" (1965)_. Finalmente aduciré mis razones por las cuales considero que la explicación funcional es un caso de explicación genuina.

\section{La explicación como conflicto}

En Diánoia 1980 (1981), J. A. Robles y yo presentamos un análisis de la explicación - afín a los de Braithwaite (1953), Kim (1964), Toulmin (1972) y Gärdenfors (1980) —según el cual, desde una perspectiva epistemológica y pragmática de ella, la raíz de toda explicación debe buscarse en un conflicto entre (por lo menos) dos creencias de un sujeto $p$, que pertenecen a niveles jerárquicos diferentes de su conjunto de creen- 
cias o a distintas teorías. Este conflicto se gesta porque hay una contradicción lógica entre las creencias involucradas $y$, cuando $p$ la percibe -bajo la forma de una situación anormal de acuerdo con sus expectativas sobre ella-, busca una explicación.

Sobre la naturaleza, la función y los componentes de la explicación afirmamos ahí mismo que: a) la palabra "explicación" designa un proceso conceptual que, cuando llega a su término, tiene como resultado que una creencia cuya verdad era ya aceptada por $p$ (debido a pruebas empíricas o fundada en alguna autoridad), se inserte en el conjunto de sus creencias racionalmente justificadas. Asi, b) la función de las explicaciones no consiste en proporcionar información sobre el mundo, sino en hacer inteligible algo ya conocido previamente -en otras palabras, la comprensión está involucrada de manera esencial en los procesos explicativos y éstos proporcionan, por añadidura, una evidencia racional en apoyo de la verdad de las proposiciones expresadas en los explananda. c) Los objetos de la explicación son proposiciones que se refieren a (presuntos) hechos y a sucesos. Estos, a su vez, son aquello sobre lo cual versan las explicaciones. ${ }^{1} d$ ) Denominamos "entidades explicativas" a algunos hechos, sucesos o leyes generales que guardan ciertas relaciones (que están conectadas de alguna manera) con las cosas sobre las cuales versan las explicaciones, determinándolas. - Las relaciones aludidas pueden ser de varios tipos, v. gr., mereológicas, de composición, causales, de consecuencia, etc. $e$ ) Se da una explicación sólo si $p$ puede ver que el (presunto) hecho o el fenómeno que intenta comprender es como es, o es de tal forma, porque mantiene ciertas relaciones determinadas con otras cosas (las entidades explicativas aludidas en $(d)$ ), cuyas proposiciones correspondientes pertenecen al conjunto de sus creencias -en esto consiste, precisamente, la evidencia racional proporcionada por la explicación. Por último, f) puesto que la comprensión por $p$ del hecho o del fenómeno que desea explicar ocurre sólo cuando reconoce que éstos son casos de un principio general, recurrir a leyes generales (conocidas o desconocidas) es también esencial a las explicaciones - que se diferencian así de las predicciones y las retrodicciones, cf. Robles, J. A. y Ponce, M., ibid., 1981, pp. 105-133. Y aducimos el siguiente ejemplo de explicación:

1 Consideramos que "proposición" designa los correlatos de nuestras actitudes proposicionales. Tiene, en consecuencia, el mismo referente que "estado de cosas" o "situación". Sin embargo, no podemos identificar estados de cosas con hechos, porque algunas veces pedimos explicaciones sobre situaciones que no son hechos, aun si (erróneamente) creemos que lo son -por ejemplo, podemos preguntar por qué el éter es perfectamente elástico y completamente rigido. Para la discusión de este problema semántico, véase Strawson (1959), Davidson (1969), Castañeda (1980), etc. 
Dos fuentes luminosas (supuestamente dos cuasares) producen aná. lisis espectrales semejantes. Si se supone que estas fuentes son, de hecho, dos cuasares, surgen serias dificultades, que se eliminan si suponemos que hay una galaxia operando como lente gravitacional -operación resultante que cabía esperar según la Teoría general de la relatividad__ entre el observador y un cuasar _...dado que dicha galaxia es la que produce dos imágenes de un único cuasar $i c j$. Chaffee 1980).

Y damos luego razón del proceso explicativo en términos de la capacidad de $p$ de atribuir probabilidades subjetivas a los objetos de sus creencias y expectativas. Al final del análisis se aclara el hecho de que las explicaciones producen una satisfacción intelectual en el sujeto. En fin, consideramos que nuestro enfoque es interesante, entre otras razones, porque relativiza las expectativas de un sujeto $p$ a un conjunto de sus creencias, lo cual nos permite comprender mejor la naturaleza de la satisfacción intelectual antes mencionada: se trata de una coherencia recuperada entre algunas de sus creencias importantes. Concluimos que comprender y aceptar una explicación concreta como una genuina explicación, depende no sólo del nivel cultural del sujeto, sino principalmente de la manera como tiene jerarquizadas sus creencias - consciente $o$ inconscientemente.

Hecha esta pequeña historia, veamos, a través de Cohen, que explicar no es informar, ni describir, ni enumerar.

\section{Cohen y la explicación}

La concepción de Cohen sobre la explicación aparece en el marco de su análisis sobre las explicaciones consecuenciales (de que forma parte la explicación funcional), de aquellas en las cuales la referencia a los resultados de un fenómeno contribuye a explicarlo. Como es consciente de que nuestras creencias sobre la naturaleza de la explicación determinan el tratamiento que puede darse a las primeras, inicia el suyo sosteniendo las tesis de que la naturaleza primordial de la explicación es aclaratoria (cf. Cohen, ibid., p. 252) y de que hay dos clases de explicación legitima, pero la pregunta "¿por qué?" inquiere por las causas (cf. ibid., p. 251). Precisa, pues, qué entiende por "explicar" y cómo usará la palabra "explicación": explicar consistiría, probablemente, en aclarar (cf. ibid., p. 252, nota 1); y, dado que toda explicación sería una respuesta o bien a preguntas “ipor qué?", o bien a preguntas “¿qué?" -what- questions, que incluirían asimismo las preguntas “cuál(es)?” y "¿cómo?”, cf. ibid., p. 251_, "explicar por qué es aclarar por qué; explicar qué, es aclarar qué, etc." (ibid., p. 252, nota 1 ). 
Para él, entonces, la pregunta "¿por qué?" inquiere siempre sobre las causas de su fenómeno, y su forma general es:

“¿Por qué es el caso que $p$ ?" [donde] " $p$ " representa una oración empírica, afirmando, por ejemplo, que el cobre conduce la electricidad o que los pájaros tienen huesos huecos... (ibid., p. 251).

La respuesta a esta clase de pregunta nos daría, paralelamente, una explicación por qué, la cual se distinguiría de las explicaciones qué por los compromisos que conlleva: en primer término, con un principio general o ley, del que dependería su carácter explicativo (cf. ibid., p. 256); en segundo término, y si la explicación es funcional, el compromiso de explicar la presencia o forma del fenómeno descrito en el explanandum, en virtud de sus resultados. Finalmente, en lo que parece una concesión no confesada al prejuicio de que las únicas explicaciones que en realidad explican son las causales, Cohen advierte que a lo largo de su análisis usará la palabra "explicación" para designar la explicación causal, no por creer que ésta sea la única genuina, sino porque es la que le interesa (cf. ibid., p. 253). ${ }^{2}$

A diferencia de la explicación por qué, en los casos siguientes se pediría una explicación qué:

1) 'Explique las reglas del ajedrez', pronunciada por alguien que desea saber cuáles son, no por qué son las que son... (ibid., p. 251).

2) 'Explique qué está sucediendo en Irlanda del Norte', de alguien que sabe solamente que algo fuera de lo común está sucediendo [alli] y que no inquiere sobre sus causas (ibid., p. 251).

3) 'Explique la estructura del ADN', si el hablante se conforma con una respuesta que no revele por qué está estructurado como está (ibid., p. 251).

4) 'Explique la función del hígado', si sólo se busca una lista de los servicios útiles que presta (ibid., p. 251).

Si reflexionamos sobre estas afirmaciones de Cohen, resulta claro que adopta una posición demasiado simplista ante el problema de la explicación: confunde informar, describir y enumerar (ejemplos (1), (3) y (4)

2 Considera, además, que "no todas las explicaciones científicas son explicaciones por qué, y no todas las explicaciones por qué son explicaciones cientificas" (ibid., p. 252): por ejemplo, "una buena explicación de la estructura del ADN es una explicación científica, pero no necesita explicar por qué alguna cosa es tal" (ibid., p. 252). Coincide en esto, parcialmente, con W. J. van der Steen, quien afirma en "Ecology, Evolution and Explanatory Patterns in Biology" (1972), que "una explicación contesta una pregunta por qué, pero no toda respuesta a cualquier pregunta por qué se cuenta como una explicación cientifica" (ibid., p. 594). 
respectivamente) con explicar. Parece sostener que el proceso explicativo consiste sólo en un esclarecimiento, y olvida que uno de sus ingredientes esenciales es la comprensión, la inteligibilidad del hecho o del fenómeno expresado en el explanandum, que se obtiene a su término. Olvida también que explicar no consiste en incrementar nuestro repertorio de datos sobre el mundo, sino que la información que puede lograrse versa primordialmente sobre la coherencia entre la verdad de lo expresado en el explanandum y nuestro sistema de creencias; que proporciona una evidencia o una justificación racional, no fáctica, de la primera. $^{3}$ Quizá Cohen se dejó llevar muy a la ligera, de una parte, por la intuición de que las explicaciones no responden sólo a preguntas sobre las causas de un fenómeno, sino que también se piden acerca de otros factores que pueden determinarlo (por ejemplo, el lugar donde acaeció, la manera como se desarrolló, etc.); ${ }^{4}$ y de otra parte, por el afán de apartarse de la posición de Hempel, autor clásico en este tema, a quien Cohen toma como contrapunto de sus propias ideas y quien sin duda es demasiado rígido en lo tocante al tipo de pregunta que puede generar una explicación. Mas si bien Hempel sostiene efectivamente que las explicaciones genuinas responden siempre a preguntas "¿por qué?" en sentido causal

-...toda explicación se lleva a cabo, en último término, por la referencia a los antecedentes causales o correlacionales... (Hempel 1959 , p. 271) -, ${ }^{5}$

señala, asimismo, que la explicación no puede consistir sólo en informar, describir o enumerar, pues

La ciencia empírica, en todas sus ramas importantes, busca no sólo describir los fenómenos del mundo de nuestra experiencia, sino también explicar o comprender su acaecimiento: se preocupa no sólo del "¿qué?", "¿cuándo?" y "¿dónde?”, sino en definitiva, y con

3 Para un examen más detenido de este asunto véase nuestro artículo ya citado, Ponce, M. y Robles J. A. 1981, pp. 110, 112-114.

4 Otros autores que han tratado este problema reconocen que no sólo la pregunta acerca de las causas conduce a la explicación. Así Peter Achinstein, en "The Object of Explanation" (1975), afirma que para identificar el objeto de la explicación hay que identificar el tipo de pregunta involucrada en cada caso, ya que pueden plantearse varios, y cada uno exige un explanans diferente, por ejemplo, "¿por que?", "¿cómo?”, "¿dónde?" (cf. Achinstein, ibid., p. 16). También Rom Harré -quien comenta este artículo de Achinstein - admite que un acontecimiento puede explicarse dando las razones de su ocurrencia o describiendo cómo sucedió (cf. Harré 1975, pp. 54-55).

5 Incluso las explicaciones probabilísticas, cuyo objetivo ideal sería, según Hempel, llegar a las causas. 
frecuencia predominantemente, del "¿por qué?" de los fenómenos que investiga (ibid., p. 271).

La dificultad estriba en que tanto la posición de Hempel como la de Cohen son discutibles: la del primero por restringir la explicación al ámbito de la búsqueda de las causas, y la del último por haber olvidado que explicar consiste no sólo en aclarar, sino en hacer inteligible algo opaco a nuestra razón. Para superar dicha dificultad necesitariamos reconciliar las tres intuiciones sobre la explicación que, en parte, la generan, a saber: (1) la que nos dice que toda pregunta explicativa es, en último término, una pregunta "¿por quée?"; (2) la que nos dice que no sólo inquirir sobre las causas puede generar una explicación y, finalmente, (3) la que nos dice que explicar no consiste sólo en informar, ni en un simple aclarar, sino en comprender. ${ }^{6}$ Esta tarea tiene sus antecedentes en Aristóteles, quien trató de armonizar las intuiciones señaladas de un modo que aún ahora parece plausible: recordemos que, según afirma, conocemos una cosa cuando captamos su por qué, su causa o principio (cf. Phys. 194b 15-20); pero habia cuatro tipos de principios explicativos; cuatro tipos de factores que podían determinar un fenómeno. $Y$ todos ellos constituían la respuesta a una pregunta "¿por quée?" Era la pregunta la que podía plantearse en sentidos diferentes, según aludiera a la causa material, a la causa formal, a la causa eficiente o a la causa final. Así, su doctrina resolvía el problema que ahora se nos presenta con urgencia: el de incluir entre la clase de preguntas que pueden generar explicaciones, a otras que no sean sólo las que inquieren por las causas. Y esto sin trivializar la explicación, como sucede con Cohen debido a que no toma en cuenta el elemento de inteligibilidad que interviene en ella.

En vista de este panorama, considero que continúa vigente la tesis sostenida en "Notas generales sobre la explicación" de que lo importante en una demanda de explicación, no es la pregunta que se haría de entrada y en un primer nivel epistemológico, sobre las causas, las consecuencias, la estructura o la localización espacio-temporal de un fenómeno. Lo que verdaderamente importa para que se inicie un proceso explicativo es que la información obtenida hasta ese momento plantee un conflicto con otras de nuestras creencias. Tal conflicto puede no estar presente en la conciencia de quien pide explicaciones; y puede adoptar la forma leve de un simple contraste o disparidad con algo que ni si-

6 El siguiente pasaje de G. Ledyard Stebbins, en Darwin to DNA, Molecules to Humanity (1982), muestra inequivocamente que los científicos también consideran (explícita o implícitamente) que explicar consiste en comprender algo: "¿Por qué los organismos han evolucionado? Contestar esta pregunta nos hará comprender [el énfasis es mio] sólo parcialmente el proceso evolutivo" (ibid., p. 24). 
quiera está bien especificado: por ejemplo, cuando se pide información sobre las reglas del ajedrez — caso (1) de Cohen_- si ha de iniciarse un proceso explicativo, la pregunta "¿cuáles?" surge en el marco de otra subyacente, como "¿por qué esas reglas y no otras?", o "¿por qué precisamente $n$ número de reglas?" En el caso de los acontecimientos en Irlanda del norte - para seguir con los ejemplos de Cohen- la pregunta sería, en el fondo, "¿por qué esos acontecimientos, en lugar de tales otros que son normales? Así, no importa tanto si la pregunta primera o aparentemente explicativa (en realidad en ese primer nivel se trata de una pregunta informativa) se expresa mediante un "¿por qué?", un "¿cómo?", un "¿dónde?", etc.; en el fondo, o mejor dicho, en el nivel de la explicación, se plantea siempre una pregunta "¿por qué?", que es la genuinamente explicativa. Esta pregunta no versa sobre las causas, el lugar o la manera de desarrollarse del fenómeno descrito en el explanandum de un argumento, sino sobre la justificación racional de éste, sobre la causa de que el fenómeno en cuestión haya ocurrido o se presente de tal modo en vez de tal otro, que podia esperarse de acuerdo con el resto de nuestras creencias. Por ello pienso que, en última instancia, Hempel tiene razón en el sentido de que las explicaciones se piden sobre las causas, pero habría que precisar que se trata de las causas de una cierta disparidad entre la verdad del explanandum (o entre el hecho expresado en el explanandum) y nuestras creencias al respecto. (Lo que constituye, quizás, el motivo por el cual se piensa que las explicaciones causales tienen preeminencia sobre las otras).

En fin, está plenamente justificado rechazar la idea de que dar explicaciones consiste en un simple aclarar (es decir, en informar, describir, enumerar, etc.); lo que sucede es que en la práctica no se puede distinguir de manera tajante cuándo un sujeto pide ser informado y cuándo desea explicarse algo. En general, estos pasos o niveles epistemológicos se entremezclan y se apoyan mutuamente, en la búsqueda de la inteligibilidad. Pero no obstante esta dificultad práctica, Sylvain Bromberger, en "An Approach to Explanation", distinguió claramente la diferencia entre lo que significan oraciones como "A explicó W a B" y "A dijo W a B". Su análisis, de gran valor teórico, tiene especial interés en este caso, pues refuerza mis afirmaciones sobre Cohen, en el sentido de que este autor trivializa la explicación al confundirla con los procesos de describir o de informar.

\section{Bromberger habla de "episodios explicativos"}

Los "episodios explicativos" serían episodios cuyo término o completamiento puede ser expresado por enunciados de la forma "A explicó W a 
B" (Bromberger 1965, p. 76) ${ }^{7}$ o, más precisamente, "A E W a B" (cf. ibid., p. 74), donde

'A' y 'B' indican lugares ocupados por expresiones mediante las cuales se menciona a alguna persona o personas (es decir, nombres propios, descripciones singulares o gente o grupos de gente específicos, pronombres personales, 'alguien', 'alguno', etc.); ' $W$ ' indica un lugar ocupado por una pregunta indirecta y ' $E$ ' indica la posición que toma 'explicar' en alguna de sus versiones temporales (ibid., p. 74).

Su objetivo es llegar a una doctrina aceptable de la explicación que señale la diferencia entre explicar y obtener información

-una perspectiva correcta sobre la naturaleza de los episodios explicativos revelaría la diferencia entre aquello que se expresa mediante enunciados de la forma 'A explicó $W$ a $B$ ' y enunciados de la forma 'A dijo W a B' [por ejemplo, 'Henrietta me explicó cuál es la altura del Empire State Building'] (ibid., p. 79),

para lo cual introduce el concepto de P-predicament -que designaré, en lo que sigue, con el término "situación p-problemática". Bromberger pide que se consideren las preguntas:

(A) ¿Cuál es la altura del monte Kilimanjaro?

(B) ¿Por qué las teteras emiten un silbido justo antes de que el agua comience a hervir? (ibid., p. 80).

Afirma que podría pedirse una explicación acerca de (B), pero no acerca de (A), porque puede estarse en una situación p-problemática respecto de (B), mas no respecto de (A). La diferencia radicaría en el tipo de ignorancia que se tenga sobre aquello que se inquiere en cada una: un sujeto podría decir con sentido "no entiendo por qué las teteras emiten un silbido justo antes que el agua comience a hervir" (ibid., p. 80), mientras que no podría decir algo similar respecto de la altura del monte

7 Con relación a los episodios explicativos, Bromberger dice que "explicar" debe considerarse un término de completamiento: pertenece a cierta clase de expresiónes que tienen un presente continuo o cuyo antepresente (como en este caso) constituye el verbo principal en respuestas a preguntas del tipo "¿qué está haciendo?" Describen episodios temporales, pero se distinguen de verbos como "correr", "hablar", "comer", etc., en que designan cierta actividad que cuando cesa, también alcanza su completamiento (por ejemplo, "llenar" y "morir") - y no, como en el caso de los verbos mencionados antes, un simple alto en la actividad de que se trate. Así, en los contextos de "A explicó W a B", "explicar" funcionaría como término de completamiento (cf. ibid., p. 75). 
Kilimanjaro (cf. ibid., p. 81). La clave, según este autor, está en que no obstante que ambas son preguntas correctas (es decir, tienen una respuesta correcta) y no obstante que el sujeto sabe (o cree saber) lo suficiente sobre dichas preguntas "como para poder eliminar un número de expresiones posibles" como respuestas correctas de ellas, tratándose de (A) tiene alguna idea aproximada de la respuesta (por ejemplo, " $3000 \mathrm{~m}$ " 0 " $2.87 \mathrm{~km}$ ") y en cambio no tiene ninguna en el caso de (B) (cf. ibid., p. 82). Así, concluye que cuando algún sujeto $A$ se halla, respecto de una pregunta $Q$ (la pregunta cuya forma en oratio obliqua aparece donde $W$ ), en la relación en la cual se hallaba respecto de (B), está en una situación p-problemática respecto de $Q$. En otras palabras, A está

en una situación p-problemática respecto de $Q$ si y sólo si, en la opinión de $\mathrm{A}, \mathrm{Q}$ puede responderse correctamente, pero $\mathrm{A}$ no puede pensar en ninguna respuesta a la cual, según la opinión de $\mathbf{A}$, no haya objeciones decisivas (ibid., p. 82).

$Y$, a continuación, destaca aquello que Hempel señalara sucintamente y que Cohen olvida: la intima relación entre explicar y comprender, que considero es clave para distinguir la explicación de otras actividades informativas. Sostiene entonces que

Todo enunciado de la forma 'estoy en una situación p-problemática respecto de $Q$ ' implica y es implicado por el correspondiente 'no entiendo $q$ ' [con ' $\mathrm{q}$ ' reemplazada por la forma en oratio obliqua de la pregunta expresada por 'Q'] (ibid., pp. 82-83). ${ }^{8}$

8 Bromberger hace notar que para estar en una situación p-problemática respecto de alguna pregunta, se requiere que el sujeto no sea completamente ignorante sobre el asunto en cuestión (cf. ibid., pp. 84-85) - lo cual coincide más o menos con una de las condiciones que $J$. A. Robles y yo señalamos como necesaria para que pudiera haber un proceso explicativo, $c f .1981$, p. 115), y eliminaria por lo menos los ejemplos (1), (3) y (4) de Cohen de la clase de preguntas que pueden generar una explicación. Bromberger ilustra todo esto con el caso de la pregunta: "¿Cómo hizo Dios a Eva, un ser humano completo, a partir de un solo hueso de Adán?". Podrían plantearse esta pregunta: (a) Rebeca, creyente poco ilustrada quien, por tanto, no ve ninguna razón para rechazar la posibilidad de dicho suceso: Dios habría tomado la costilla de Adán $\mathrm{y}$, rompiéndola en varias piezas según las partes planeadas para Eva, las habría moldeado, ensamblado y, finalmente, animado. (b) Sam, también creyente, pero conocedor de teorías científicas actuales, quien por una parte, no puede aceptar la respuesta de Rebeca y, por otra, tampoco puede pensar en alguna alternativa aceptable (ibid., p. 84). Y, por último, (c) Max el ateo, quien rechazaría de entrada la pregunta. Así, "Sam estaria en lo cierto al decir que él mismo está en una situación p-problemática respecto de la pregunta... y tendria sentido para Max... decir que Sam está en una situación p-problemática respecto de ella, aun cuando Max no podría decir correctamente que Sam no entiende cómo Dios creó a Eva [porque decir "A no entiende q' implicaría que la pregunta expresada por ' $q$ ' es una pregunta correcta, lo cual Max no cree, y esto mismo no es implicado por 'A está en una situación p-problemá. 
Bromberger puede entonces decir que las condiciones de verdad de los enunciados de la forma "A explicó W a B" consisten en que haya ocurrido un episodio:

(a) en cuyo tiempo, el tutor [-A-] conoce la respuesta correcta de la pregunta;

(b) al comienzo del cual, el tutorando [-_-B-] estaba en una situación p-problemática respecto de la pregunta [esto implica, a la vez, que la pregunta debe tener una respuesta correcta];

(c) al comienzo del cual, el tutorando piensa que él mismo se halla en una situación p-problemática respecto de la pregunta;

(d) durante el cual, el tutor sabe, cree, o cuando menos supone, que al comienzo del episodio el tutorando estaba en una situación p-problemática respecto de la pregunta;

(e) en el curso del cual, el tutor presenta los hechos que, en su opinión, el tutorando debe aprender para conocer la respuesta correcta a la pregunta;

(f) al término del cual, todos los hechos mencionados en (e) han sido presentados por el tutor al tutorando;

(g) al término del cual, el tutorando conoce la respuesta correcta, y la conoce como resultado de, y en virtud de aquello que le ha dicho el tutor en el curso del episodio (ibid., pp. 85-86). ${ }^{\circ}$

$\mathrm{Y}$, dadas estas condiciones de verdad, resulta claro por qué los casos en que se dice solamente algo que no se sabía antes, por ejemplo, la altura del Empire State Building, no constituyen respuestas a preguntas que puedan generar explicaciones, es decir, a preguntas respecto de las cuales algún sujeto esté en una situación p-problemática (cf. ibid., p. 87).10

tica respecto de q' (Cf, ibid., p. 83)]. Pero Sam, Max o Rebeca... estarian equivocados si dijesen que Rebeca está en una situación p-problemática respecto de la pregunta, puesto que ella puede concebir por lo menos una posibilidad que no necesita rechazar. Sin embargo, Sam estaria en lo cierto al decir que ella no entiende cómo Dios creó a Eva. [Y] ninguno deberá decir que Max se halla en una situación p-problemática respecto de la pregunta" (ibid., p. 84).

- Bromberger señala que no todas las cláusulas expuestas representan condiciones necesarias para que se dé un episodio explicativo - por ejemplo, (b), (g), (c) y (d) (cf. ibid., p. 86). Pero si eliminásemos todas estas cláusulas, no tendríamos siquiera un conjunto de condiciones suficientes, lo cual, en su opinión, sí ha proporcionado mediante (a) - (g), aunque sólo puede apoyar esto en el hecho de que aún no se ha podido aducir ningún ejemplo que satisfaga estas cláusulas y no constituya un episodio explicativo (cf. ibid., p. 87).

10 Añade que la exigencia de que se esté en una situación p-problemática para que pueda generarse un episodio explicativo, eliminaria de entrada ciertas preguntas como posibles fuentes de explicación: por ejemplo: (a) preguntas indirectas que comienzas con "sea que", "ora", cuyas respuestas correctas son si o no, ya que ningún tutorando estaría en dicha situación respecto de ellas; (b) preguntas indirectas que comienzan 
Y también resulta claro por qué las preguntas (1), (3) y (4) de Cohen no pueden generar una explicación. Sus respuestas carecerían de otro rasgo esencial de ésta: el de proporcionar una evidencia o justificación racional (cf. Hempel 1965, pp. 367-368) de la verdad del explanandum, efecto aludido por Bromberger cuando dice que las oraciones de la forma "A explica $W$ a B" reportan

episodios en los cuales el tutor convierte a alguien que hubiera podido decir con verdad: 'No entiendo $W$ ', en alguien en la posición de afirmar 'Sé que W' (ibid., p. 88).

\section{La explicación funcional como explicación genuina}

Resta ahora preguntarnos si, puesto que en todo proceso explicativo la proposición (sobre un hecho o sobre un fenómeno) expresada por el explanandum (o por " $Q$ " según Bromberger) queda justificada racionalmente -es decir, se vuelve consistente o coherente con el resto de nuestras creencias-, las explicaciones funcionales son genuinas explicaciones, desde esta perspectiva. Mi respuesta es que sí, porque proporcionan, sin duda, esa evidencia o justificación racional. Ello puede verse en forma clarísima en la reconstrucción que hace E. Nagel de un enunciado funcional típico, y que transcribiré ahora (aunque la haya citado o mencionado anteriormente, por ejemplo, en Diánoia 1981 (1982)), porque ilustra bien este punto:

En La estructura de la ciencia (1961), Nagel reformula el enunciado

'La función de la clorofila en las plantas es permitir que éstas realicen la fotosntesis...' (Nagel, ibid., p. 403).

cuya forma general es

'La función de $A$ en un sistema $S$ de organización $C$, es permitir que $S$ realice el proceso $P$ en un medio ambiente $E^{\prime}$ (ibid., p. 403),

\section{como el argumento}

con “¿cuál es la altura de ...?”, “¿Cuál es el peso de ...?”, “¿Cuál es la distancia entre...?, es decir, "preguntas que piden una respuesta expresable en forma de un numero y una unidad de medida" (ibid., p. 89) porque generalmente el tutorando tampoco puede estar en una situación p-problemática ante ellas. $\mathrm{Y}$ (c) preguntas indirectas que comienzan con "cuál", debido a que las oraciones interrogativas que comienzan con esta palabra sólo formularían una pregunta si están asociadas con un conjunto específico de alternativas que incluyen la respuesta correcta (en caso de que haya alguna). Así, el tutorando tampoco podría estar en una situación p-problemática respecto de ellas (cf. ibid., p. 90) -lo cual eliminarla los ejemplos mencionados de Cohen, que comienzan precisamente con la palabra "cuál (es)"... 
Todo sistema $S$ de organización $C$ y en un ambiente $E$, realiza el proceso $P$; si $S$ de organización $C$ y en un ambiente $E$ no tiene $A$, entonces $S$ no realiza $P$;

Por tanto, $\mathrm{S}$ de organización $\mathrm{C}$ debe [el énfasis es mío] tener A (ibid., p. 403).

Podemos ver que la fuerza de una explicación de este tipo no reside en el descubrimiento de ningún dato nuevo sobre el mundo, ni en señalar las causas de la presencia o de la forma de la entidad funcional -A - en un sistema determinado, sino en la inteligibilidad que adquiere una proposición -en este caso, si $S$ realiza $P$, entonces debe tener $A$ para nosotros: al término del proceso explicativo sabemos que es cierta, pero con un tipo de certeza que no podrian proporcionar ni la experiencia ni una opinión autorizada ni el consenso de la mayoría ni ningún otro medio por el cual nos hubiéramos enterado previamente de su verdad. La palabra "debe" en el argumento de Nagel tiene la fuerza de la justificación o evidencia racional, aludida también por Aristóteles cuando, a propósito de lo que es explicar, dice que

comenzamos asombrándonos de que la diagonal del cuadrado sea inconmensurable respecto del lado; debemos terminar en un estado en el que deberíamos asombrarnos si fuese de otra manera (Met. 983a 11-18).

En esto consiste la inteligibilidad que proporciona una explicación genuina y que, como hemos visto, proporcionan asimismo las explicaciones funcionales.

\section{BIBLIOGRAFIA}

Achinstein, Peter (1975), “The Object of Explanation”. En Explanation, ed. por Stephan Körner. Basil Blackwell, Great Britain, pp. 1-45.

Aristóteles, Physical (trad. R. P. Hardie y R. K. Gaye), The Basic Works of Aristotle, Random House, New York, 1941.

Metaphysica '(trad. W. D. Ross), ibid.

Braithwaite, Richard B. (1953), Scientific Explanation. Reimpreso por Harper \& Brothers, New York, 1960.

Bromberger, Syvain (1965), “An Approach to Explanation". Analytical Philosophy, ed. por R. J. Butler, Basil Blackwell, Great Britain, II, pp. 72-105. Castañeda, Héctor-Neri (1980), "Reference, Reality and Perceptual Fields". Proceedings and Addresses of the American Philosophical Association, 53, 6, pp. 763-823. 
Cohen, G. A. (1978), Karl Marx's Theory of History, Clarendon Press, Oxford, Great Britain.

Chaffee, Frederic H. Jr. (1980), "The Discovery of a Gravitational Lens". En Scientific American, 243, 5, pp. 60-68.

Davidson, Donald (1969), "The Individuation of Events". En Essays in Honor of Carl G. Hempel, ed. por Nicholas Rescher et al., D .Reidel Publ. Co., Dordrecht, Holland, pp. 216-234.

Gärdenfors, Peter (1980), "A Pragmatic Approach to Explanation". Philosophy of Science, E.U.A., 47, 3, pp. 404-423.

Harré, Rom (1975), "Comment". En Explanation, ed. por Stephan Körner, Basil Blackwell, Great Britain, pp. 54-66.

Hempel, C. G. (1959), "The Logic of Functional Analysis". En Gross, Llewellyn (eds.), Symposium on Sociological Theory, Harper \& Row, New York.

Kim, Jaegwon (1964), "Inference, Explanation, and Prediction". Journal of Philosophy, 61, pp. 360-368.

Nagel, Ernest (1961), La estructura de la ciencia, Paidós, Buenos Aires, 1968.

Ponce, Margarita y Robles, José A. (1981), "Notas generales sobre la expicación". Diánoia 1980, F.C.E., México, pp. 105-133.

Ponce, Margarita (1982), "Las explicaciones teleológicas y el modelo nomoló. gico deductivo". Diánoia 1981, F.C.E., México, pp. 186-206.

Stebbins, Ledyard G. (1982), Darwin to DNA, Molecules to Humanity, W. H. Freeman \& Co., E.U.A.

Steen, W. J. van der (1972), "Ecology, Evolution and Explanatory Patterns in Biology". Journal of Theorethical Biology, 36, pp. 593-616.

Toulmin, Stephen (1972), Human Understanding, Vol. I., Oxford University Press, Great Britain. 